\title{
Energy harvesting of nonlinear damping system under time delayed feedback gain
}

\author{
A. Bichri ${ }^{1}$, I. $\operatorname{Kirrou}^{2,3, a}$, M. Belhaq ${ }^{2}$ \\ 1 Faculty of Science and Technology-Errachidia, University Moulay Ismail, Morocco \\ 2 Laboratory of Renewable Energy and Structural Dynamics, University Hassan II-Casablanca, Morocco \\ 3 Laboratory of Engineering Science, ESTEM Research Center, Casablanca, Morocco
}

\begin{abstract}
This paper presents the application of delayed feedback velocity for optimizing the harvested power in cubic nonlinear damper system. We consider a harvester consisting of a nonlinear single degree of freedom system (spring-masse-damper) subjected to a base excitation near the primary resonance. Analytical investigation using the multiple scales method is performed to obtain approximation of the amplitude response. This amplitude can be used to extract the average power. Results show that for appropriate values of the feedback gain, energy harvesting is more efficient at resonance compared to the cubic nonlinear damper system without time delay.
\end{abstract}

\section{Introduction}

This paper focuses on energy harvesting $(\mathrm{EH})$ performance extracted from vibrations provided by ambient energy as, for instance, wind induced vibrations and sailing ships oscillations. To increase the frequency range where EH operates, various models have been suggested [2-4].

In the case where the harvester operates within the linear regime, the maximum power is generated when the system is excited at resonance $[5,6]$. To overcome this limitation, different alternative approaches have been explored by introducing nonlinear components into the system. In some systems $[7,8]$ the nonlinear stiffness is accompanied by nonlinear damping.

On the other hand, to enhance the energy harvesting performance a new concept based on delayed feedback control has been performed. A recent work reported on this issue considered a delayed van der Pol oscillator with modulated delay amplitude [4]. It was shown that modulating the delay amplitude may produce large amplitude quasiperiodic vibrations in self-excited oscillators.

In the present work, we study the $\mathrm{EH}$ in a cubic nonlinear damper system using a delayed feedback velocity. The harvester consists of a nonlinear single degree of freedom system (spring-masse-damper) subjected to a base excitation near the primary resonance. This study extends the results obtained in the case without the time delay reported in [1].

The rest of the manuscript is organized as follows: In section 2, we present the mathematical model of the energy harvesting system. In section 3 we perform the method of multiple scales to obtain the amplitude-phase response near the primary resonance. The effect of delay amplitude on the frequency response and the average power is reported and discussed. Section 4 concludes the work.

\section{The dynamic model and perturbation analysis}

We consider a harvester device consisting of a nonlinear single degree of freedom system (spring-masse-damper) subjected to a base excitation near the primary resonance. The quantities $F_{K}, F_{d p}$ and $F_{d}$ are, respectively, the nonlinear restoring force, the nonlinear damping force and the delay feedback in the velocity. The schematic model of the system is shown in Fig. 1 and the equation of motion can be written in the form

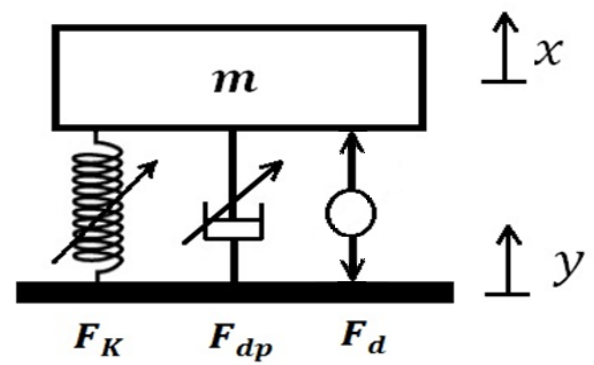

Fig. 1. Single degree of freedom, base excited, energy harvesting system.

$$
\begin{array}{r}
m \ddot{\bar{z}}(t)+c_{1} \dot{\bar{z}}(t)+c_{3} \dot{\bar{z}}^{3}(t)+K_{1} \bar{z}(t)+K_{3} \bar{z}^{3}(t)=-m \ddot{y}(t) \\
-\alpha_{d} \dot{\bar{z}}(t-\bar{T})
\end{array}
$$

where the overdot represents a derivative with respect to time $t$. The variable $\bar{z}$ represents the relative displacement of the mass $m, c_{1}$ and $c_{3}$ are damping coefficients, $k_{1}$ and $k_{3}$ are, respectively, the linear and nonlinear stiffness of the restoring force, $\alpha_{d}$ is the delay amplitude, $\bar{T}$ is the time delay and $y(t)=Y \cos \Omega t$ is the harmonic base motion. The relative normal displacement is given by

$$
\bar{z}(t)=x(t)-y(t)
$$


To obtain a dimensionless form of Eq. (1), we introduce the following nondimensional parameters: $\xi_{1}=\frac{c_{1}}{2 m \omega_{0}}, \xi_{3}=$ $\frac{c_{3} \omega_{0} Y^{2}}{m}, \gamma=\frac{K_{3} Y^{2}}{m \omega_{0}^{2}}, \lambda_{d}=\frac{\alpha_{d}}{m \omega_{0}}, \omega_{0}=\sqrt{\frac{K_{1}}{m}}, T=\omega_{0} \bar{T}, \tau=\omega_{0} t$, $\omega=\frac{\Omega}{\omega_{0}}, z=\frac{\bar{z}}{Y}, \alpha_{1}=2 \xi_{1}, \alpha_{2}=\xi_{2}$. The dimensionless equation of motion takes the form

$$
\ddot{z}+\alpha_{1} \dot{z}+\alpha_{2} \dot{z}^{3}+z+\gamma z^{3}=\omega^{2} \cos (\omega \tau)-\lambda_{d} \dot{z}(\tau-T)
$$

To analyze the effect of the time-delayed feedback on the frequency response near the primary resonance, we use the method of multiples scales $[10,11]$. To this end, we express the resonance condition by introducing a detuning parameter $\sigma$ according to $\Omega_{0}^{2}=\omega^{2}+\varepsilon \sigma$ and $\Omega_{0}^{2}=1$. Introducing a small parameter $\varepsilon$, the equation of motion reads

$$
\begin{array}{r}
\ddot{z}+\omega^{2} z=\varepsilon\left(-\sigma z-\alpha_{1} \dot{z}-\alpha_{2} \dot{z}^{3}+\omega^{2} \cos (\omega \tau)-\right. \\
\left.\lambda_{d} \dot{z}(\tau-T)\right)-\varepsilon^{2} \gamma z^{3}
\end{array}
$$

To ultimately solve Eq. (4), steady-state solution are expanded as

$$
\begin{aligned}
z(\tau)=z_{0}\left(\tau_{0}, \tau_{1}, \tau_{2}\right)+\varepsilon z_{1}\left(\tau_{0}, \tau_{1}, \tau_{2}\right)+\varepsilon^{2} z_{2}\left(\tau_{0},\right. & \left.\tau_{1}, \tau_{2}\right) \\
& +O\left(\varepsilon^{3}\right)(5)
\end{aligned}
$$

where $T_{0}=\tau, T_{1}=\epsilon \tau$ and $T_{2}=\epsilon^{2} \tau$. In terms of the variables $T_{i}(i=0,1,2)$, the time derivatives become $\frac{d}{d \tau}=$ $D_{0}+\varepsilon D_{1}+\varepsilon^{2} D_{2}+O\left(\varepsilon^{3}\right)$ and $\frac{d^{2}}{d \tau^{2}}=D_{0}^{2}+2 \varepsilon D_{0} D_{1}+\varepsilon^{2} D_{1}^{2}+$ $2 \varepsilon^{2} D_{0} D_{2}+O\left(\varepsilon^{3}\right)$, where $D_{i}=\frac{\partial}{\partial T_{i}}$. Substituting (5) into (4), equating terms of different order of $\varepsilon$, resolving and eliminating the secular terms, we obtain the following slow flow modulation equation of the amplitude and the phase:

$$
\left\{\begin{array}{l}
\frac{d r}{d \tau}=h \sin \theta+A r+C r^{3} \\
r \frac{d \theta}{d \tau}=h \cos \theta+B r+D r^{3}
\end{array}\right.
$$

Equilibria of this slow flow, corresponding to periodic solutions of Eq. (4), are determined by setting $\frac{d r}{d t}=\frac{d \theta}{d t}=0$. This leads to the amplitude-frequency response equation

$$
\begin{array}{r}
\left(D^{2}+C^{2}\right) r^{6}+2(A C+B D) r^{4}+\left(A^{2}+B^{2}\right) r^{2}- \\
h^{2}=0
\end{array}
$$

where $A=\frac{\alpha_{1}}{2}-\frac{\lambda_{d}}{2} \cos \phi, B=\frac{\sigma}{2 \omega}+\frac{\lambda_{d}}{2} \sin \phi, C=\frac{3 \alpha_{2} \omega^{2}}{8}$, $D=\frac{3 \gamma}{8 \omega}, h=-\frac{\omega}{2}, \phi=\omega T$.

The average power is obtained by averaging over one period of the excitation. This leads to

$$
P_{\text {gen }}=\frac{\omega}{2 \pi} \int_{0}^{\frac{2 \pi}{\omega}}\left(\alpha_{1} \dot{z}+\alpha_{3} \dot{z}^{3}\right) \dot{z} d \tau
$$

Using Eq. (8), we obtain the generated average power

$$
P_{g e n}^{a v}=\frac{\alpha_{1} \omega^{2} r^{2}}{2}+\frac{3 \alpha_{3} \omega^{4} r^{4}}{8}
$$

The power required for the active control action is given by

$$
P_{a c t}=\frac{\omega}{2 \pi} \int_{0}^{\frac{2 \pi}{\omega}} \lambda_{d} \dot{z}(t-\tau) \dot{z} d \tau
$$

Then, the consumed average power is given by

$$
P_{a c t}^{a v}=\frac{\lambda_{d} \omega^{2} r^{2}}{2} \cos \phi
$$

where $\psi=\omega \tau+\theta$ and $\phi=\omega T$.

The harvested (or net) average power defined as the difference of generated and consumed average powers reads

$$
P_{\text {harv }}^{a v}=P_{g e n}^{a v}-P_{a c t}^{a v}
$$

\section{Main results}

The influence of different system parameters on the dynamic response and the maximum average output powers is examined. We fix the damping parameters $\alpha_{1}=0.25$, $\alpha_{2}=0.025$ and $\gamma=0.45$.

The frequency-response curve, as expressed by Eq. (7), is depicted in Fig. 2 for increasing delay amplitude $\lambda_{d}$. The solid lines correspond to stable branches while the dashed line corresponds to the unstable one. The plots indicate that as the delay amplitude is increased the maximum deflection displays an increasing in the hardening nonlinear response and shift toward higher frequencies.

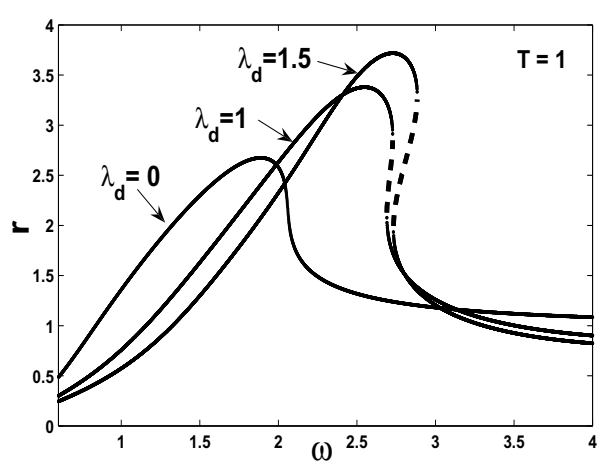

Fig. 2. Amplitude-frequency response versus $\omega$ for different values of the delay amplitude $\lambda_{d}$.

Figure. 3 illustrates the variation of the amplitude response versus the delay amplitude $\lambda_{d}$ for a fixed values of the time delay $T$ and the excitation frequency $\omega$. Analytical approximations (solid lines) are compared to numerical simulations (circles). It can be seen that increasing the amplitude $\lambda_{d}$ causes an increase in the amplitude response. Figure. 4 represents the generated average power response versus the delay amplitude $\lambda_{d}$ for a fixed value of $T$ and $\omega$. The figure shows an increase of the output power by increasing $\lambda_{d}$.

Figure. 5 depicts the variation of the average powers quantities with respect to the delay amplitude $\lambda_{d}$ for a given values of $\omega$ and $T$. It is observed that the harvested average power increases in a certain range of $\lambda_{d}$.

\section{Conclusions}

The application of delayed feedback velocity for optimizing the harvested power in cubic nonlinear damper system 


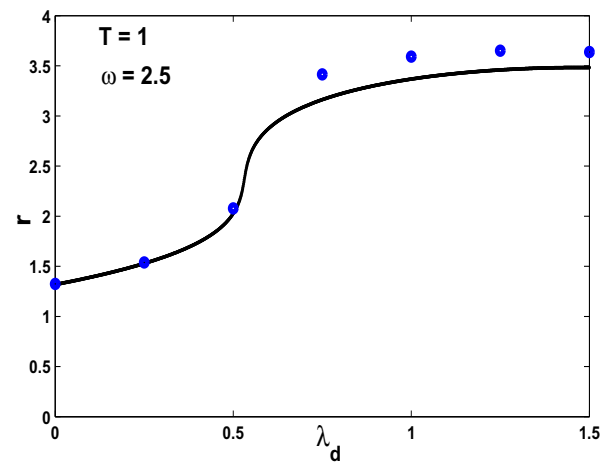

Fig. 3. Amplitude response versus $\lambda_{d}$ for $T=1$ and $\omega=2.5$; Solid lines: analytical approximation; circle: numerical simulation.

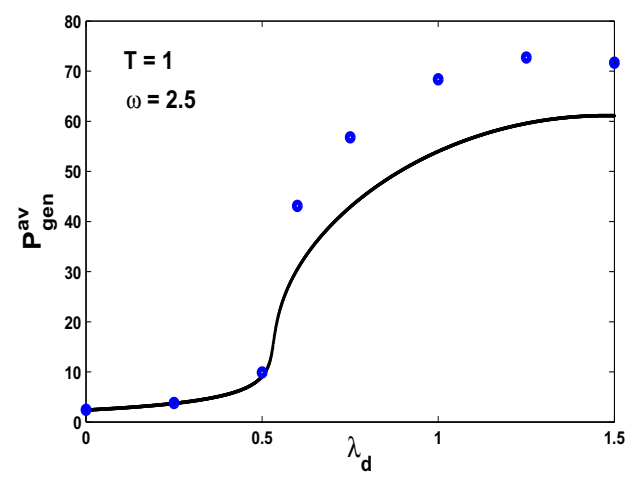

Fig. 4. Generated average power versus $\lambda_{d}$ for $T=1$ and $\omega=2.5$; Solid lines: analytical approximation; circle: numerical simulation.

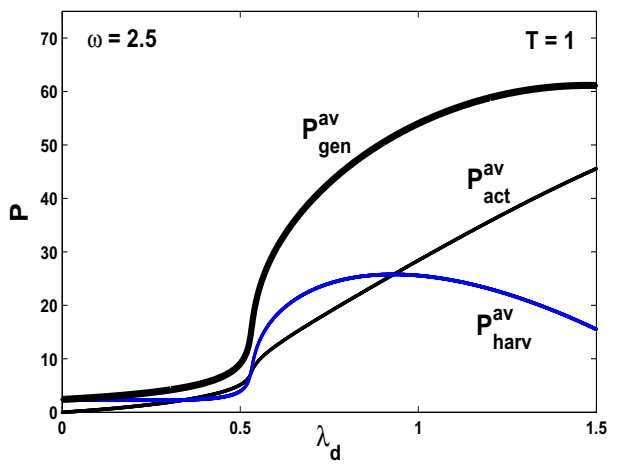

Fig. 5. Average powers quantities versus the delay amplitude $\lambda_{d}$ for $T=1$ and $\omega=2.5$.

is presented. The harvester device consists of a nonlinear single degree of freedom system (spring-masse-damper) subjected to a base excitation is considered near the primary resonance. Analytical investigation using the multiple scales method was performed to obtain approximation of the amplitude response. This amplitude is used to obtain the evolution of the extract average power as the delayed feedback gain is increased. Results shown that in the cubic nonlinear damper system, time delay introduced in the velocity enhance significantly the performance of the net energy harvesting.

\section{References}

[1] M.G., Tehrani, S.J., Elliott, Exetending the dynamic range of an energy harvester using nonlinear damping, Journal of Sound and Vibration 333: 23-629, (2014).

[2] T.S., Osorio, M.F., Daqaq, Energy harvesting under excitations of time varying frequency, Journal of Sound and Vibration, 329: 2497-2515, (2010).

[3] A., Erturk, D.J., Inman, Broadband piezoelectric power generation on high-energy orbits of the bistable Duffing oscillator with electromechanical coupling, Journal of Sound and Vibration, 330: 2339-2353, (2011).

[4] M., Hamdi, M., Belhaq, Energy from harvesting from quasi-periodic vibrations, Nonlinear dynamics, 1-13, (2016). DOI 10.1007/s11071-016-2668-6.

[5] N.G., Stephen, On energy harvesting from ambient vibration, Journal of Sound and Vibration, 293: 409-425 (2006).

[6] H.A., Sodano, D.J., Inman, G., Park, Generation and storage of electricity from power harvesting devices, Journal of Intelligent Material Systems and Structures 16: 67-75, (2005).

[7] B.P., Mann, N., Sims, Using nonlinearity to improve the performance of vibration-based energy harvesting devices, proceedings of the 7th European Conference on Structural Dynamics, Southampton July 2008.

[8] B.P., Mann, N.D., Sims, Energy harvesting from the nonlinear oscillations of magnetic levitation, Journal of Sound and Vibration 319: 515-530, (2009).

[10] A.H., Nayfeh, D.T., Mook, Nonlinear Oscillations. Wiley, New York (1979).

[11] A.H., Nayfeh, Introduction to Perturbation Techniques. New York: Wiley (1981). 